\title{
Plasma neurofilament light chain concentration is increased in anorexia nervosa
}

Ida A. K. Nilsson (1) ${ }^{1,2,3}$, Vincent Millischer $\mathbb{B}^{1,2}$, Virginija Danylaité Karrenbauer ${ }^{4,5}$, Anders Juréus ${ }^{6}$, Alireza M. Salehi', Claes Norring $\mathbb{B}^{7,8}$, Yvonne von Hausswolff-Juhlin ${ }^{7,8,9}$, Martin Schalling ${ }^{1,2}$, Kaj Blennow ${ }^{10,11}$, Cynthia M. Bullik ${ }^{3,6,12,13}$, Henrik Zetterberg ${ }^{10,11,14,15}$ and Mikael Landén 6,10

\begin{abstract}
Anorexia nervosa (AN) is a severe psychiatric disorder with high mortality and, to a large extent, unknown pathophysiology. Structural brain differences, such as global or focal reductions in grey or white matter volumes, as well as enlargement of the sulci and the ventricles, have repeatedly been observed in individuals with AN. However, many of the documented aberrances normalize with weight recovery, even though some studies show enduring changes. To further explore whether AN is associated with neuronal damage, we analysed the levels of neurofilament light chain (NfL), a marker reflecting ongoing neuronal injury, in plasma samples from females with AN, females recovered from AN (AN-REC) and normal-weight age-matched female controls (CTRLS). We detected significantly increased plasma levels of NfL in AN vs CTRLS $\left(\right.$ median $_{\mathrm{AN}}=15.6 \mathrm{pg} / \mathrm{ml}, I Q R_{\mathrm{AN}}=12.1-21.3$, median $_{\mathrm{CTRL}}=9.3 \mathrm{pg} / \mathrm{ml}$, $I Q R_{C T R L}=6.4-12.9$, and $\left.p<0.0001\right)$, AN vs AN-REC (median ${ }_{\text {AN-REC }}=11.1 \mathrm{pg} / \mathrm{ml}, I \mathrm{QQR}$ AN-REC $=8.6-15.5$, and $\left.p<0.0001\right)$, and AN-REC vs CTRLS ( $p=0.004$ ). The plasma levels of NfL are negatively associated with BMl overall samples ( $\beta$ ( \pm se) $=-0.62 \pm 0.087$ and $p=6.9 \cdot 10^{-12}$ ). This indicates that AN is associated with neuronal damage that partially normalizes with weight recovery. Further studies are needed to determine which brain areas are affected, and potential long-term sequelae.
\end{abstract}

\section{Introduction}

Anorexia nervosa (AN) is a psychiatric disorder characterized by persistent restriction of food intake resulting in significantly low body weight, combined with fear of gaining weight or behaviours that interfere with weight gain, and body image distortion ${ }^{1,2}$. The disorder affects $\sim 1 \%$ of females and $0.1 \%$ of males, and an $\sim 10 \%$ lethality makes it the most lethal psychiatric disorder ${ }^{3-5}$. On top of that, relapse rates and treatment failures are very

\footnotetext{
Correspondence: Ida A. K. Nilsson (ida.nilsson@ki.se)

${ }^{1}$ Department of Molecular Medicine and Surgery, Karolinska Institutet, Stockholm, Sweden

${ }^{2}$ Center for Molecular Medicine, Karolinska University Hospital, Stockholm, Sweden

Full list of author information is available at the end of the article.

These authors contributed equally: Vincent Millischer, Virginija Danylaité

Karrenbauer
}

common $^{6}$. The pathoetiology has not been clarified, even though interactions among genetic, environmental, and neurobiological factors clearly contribute ${ }^{1}$. Twin studies have identified a strong genetic contribution, i.e., $58-70 \%$ of variance in liability is due to additive genetic factors ${ }^{5,7,8}$. Structural brain differences, most commonly global or focal reductions in grey matter or white matter (WM) volume, and sulci or ventricular enlargement, have repeatedly been shown in individuals with $\mathrm{AN}^{9-11}$. However, imaging studies of AN patients also exist that show no structural brain changes, particularly with regards to $\mathrm{WM}^{12,13}$. Importantly, structural changes reversed upon weight restoration in several studies ${ }^{12,14-20}$, while a few documented enduring changes ${ }^{13,21,22}$. This heterogeneity in results encourages to evaluate potential brain atrophy and neurodegeneration by complementary 
methods. Moreover, it is not known if the structural brain changes seen in AN are related to degeneration of brain cells or merely changes in fluids, as discussed by Ehrlich and et al. $^{23}$. Combining results from well-designed imaging studies with analyses of markers in blood or cerebrospinal fluid (CSF) could help further explicate the neurobiology of AN.

In the present study, we hypothesize that neuronal injury and/or degeneration is involved in the pathophysiology of AN. To explore this, we measured the levels of neurofilament light chain (NfL), a marker reflecting ongoing neuronal damage, which can be reliably measured in serum, plasma or $\mathrm{CSF}^{24-26}$. We analysed plasma samples in a discovery cohort and a replication cohort, each stratified by three groups: (i) females with AN, (ii) females recovered from AN (AN-REC), and (iii) normalweight age-matched female controls (CTRLS).

\section{Materials and methods \\ Participants}

For the discovery cohort, females with $\mathrm{AN}(n=12)$ and weight-recovered females with a history of AN (AN-REC, $n=11$ ) were recruited from Stockholm Centre for Eating Disorders (SCÄ). The general inclusion criteria for the AN group were female patients, at least 18 years old, meeting the DSM-IV criteria for $\mathrm{AN}^{2}$, and with at least 5 years since $A N$ onset. AN-REC inclusion required weight recovery $(B M I>18)$ for at least 1 year. Normal-weight female controls (CTRLS, $n=12$ ), without any own or family history of eating disorders were recruited via advertisements at Karolinska Institutet and internet (ki.se and studentkaninen.se).

For the replication cohort, participants were identified from the Swedish cohort of the Anorexia Nervosa Genetics Initiative (ANGI-SE), for details on the recruitment procedure see ref. ${ }^{27}$. The general inclusion criteria for the AN replication group was female patients, at least 18 years old, meeting the DSM-IV criteria for $\mathrm{AN}^{2}$, with at least 1 year since AN onset $(n=112)$. For AN-REC, weight restoration $(\mathrm{BMI}>20)$, no eating disorder behaviours for at least a year, and being within $1 \mathrm{SD}$ of the mean for eating disorders cognitions (self-reports), were the inclusion criteria $(n=114)$. Age-matched normalweight female controls had no history of disordered eating behaviour (CTRLS, $n=113$ ). See Table 1 for further information on the study participants.

The study was approved by the Regional Ethics Review Board in Stockholm. All participants provided oral and written informed consent to participate.

\section{Blood sampling and the NfL assay}

For the discovery cohort venous blood was collected into vacutainer tubes including anticoagulant (sodium citrate) at SCÄ and processed within $2 \mathrm{~h}$. For the
Table 1 Demographic and clinical characteristics of the study participants

\begin{tabular}{|c|c|c|c|}
\hline Characteristics & AN & AN-REC & CTRL \\
\hline \multicolumn{4}{|l|}{ Discovery cohort } \\
\hline$n$ & 12 & 11 & 12 \\
\hline Females (\%) & 100 & 100 & 100 \\
\hline Age (years) & 31.0 & 28.0 & 27.5 \\
\hline (median [IQR]) & $(28.0-44.5)$ & $(24.0-34.0)$ & $(24.0-31.3)$ \\
\hline BMI $\left(\mathrm{kg} / \mathrm{m}^{2}\right)$ & 14.8 & 19.8 & 24.1 \\
\hline (median [IQR]) & $(13.4-16.7)$ & (18.8-21.8) & $(21.4-26.2)$ \\
\hline $\begin{array}{l}\text { Years since AN onset } \\
\text { (median }[\mathrm{IQR}] \text { ) }\end{array}$ & $\begin{array}{l}17.8 \\
(10.5-27.3)\end{array}$ & No info & - \\
\hline \multicolumn{4}{|l|}{ Replication cohort } \\
\hline$n$ & 112 & 114 & 113 \\
\hline Females (\%) & 100 & 100 & 100 \\
\hline $\begin{array}{c}\text { Age (years) } \\
\text { (median [IQR]) }\end{array}$ & $\begin{array}{l}26.0 \\
(24.0-31.0)\end{array}$ & $\begin{array}{l}26.0 \\
(24.0-31.0)\end{array}$ & $\begin{array}{l}26.0 \\
(24.0-31.0)\end{array}$ \\
\hline $\begin{array}{r}\text { BMI }\left(\mathrm{kg} / \mathrm{m}^{2}\right) \\
\text { (median [lQR]) }\end{array}$ & $\begin{array}{l}16.0 \\
(15.0-17.0)\end{array}$ & $\begin{array}{l}22.0 \\
(21.0-24.3)\end{array}$ & $\begin{array}{l}23.0 \\
(22.0-26.0)\end{array}$ \\
\hline $\begin{array}{l}\text { Years since AN onset } \\
\text { (median }[\mathrm{IQR}] \text { ) }\end{array}$ & $\begin{array}{l}10.0 \\
(6.0-14.3)\end{array}$ & $\begin{array}{l}10.0 \\
(6.0-14.0)\end{array}$ & - \\
\hline
\end{tabular}

$A N$ anorexia nervosa, $A N-R E C$ recovered from anorexia nervosa, CTRL healthy controls, $I Q R$ interquartile range

replication cohort venous blood was collected into EDTA-tubes at the nearest hospital, mailed to Karolinska Institutet Biobank and processed upon arrival. After centrifugation, plasma samples were stored at $-80^{\circ} \mathrm{C}$. Samples were transported on dry ice to the Clinical Neurochemistry laboratory at Sahlgrenska University Hospital where NfL concentration was measured using an in-house Single molecule array method as previously described in detail ${ }^{28}$. The measurements were performed in one round of experiments each for the discovery and replication cohorts. The intra- and interassay coefficients of variation were below $7 \%$ for QC samples with NfL concentrations of $20.4 \mathrm{pg} / \mathrm{ml}$ and $64 \mathrm{pg} / \mathrm{ml}$, respectively.

\section{Statistical analyses}

Demographic and clinical characteristics are presented using descriptive statistics.

Linear regression was used to analyse group differences (corrected for age) and to analyse the effects of age, BMI, and years since AN onset on plasma concentrations of NfL.

Statistical analyses were conducted using $\mathrm{R}$ programming language (including packages emmeans and multcomp ref. ${ }^{29}$ ). Graphs were built using ggplot2 ref. ${ }^{30}$. $P$-values $<0.05$ were considered statistically significant. 


\section{Results}

Demographic and clinical characteristics of the study population are summarized in Table 1. As has been shown previously ${ }^{31}$, age at sampling was positively associated with plasma NfL levels, in both the discovery and the replication sample (Discovery sample: $\beta_{\text {discovery }}( \pm \mathrm{se}$ ) $=0.45 \pm 0.22$ and $p=0.048$; Replication sample: $\beta_{\text {replication }}$ $( \pm$ se $)=0.37 \pm 0.087$ and $p=2.7 \cdot 10^{-5}$ ) (Fig. 1 ). We therefore corrected for age in all subsequent statistical analyses.

In both the discovery and replication sample, plasma levels of NfL were significantly higher in AN compared both with AN-REC (Discovery sample: $\operatorname{median}_{\mathrm{AN}}=$ $24.8 \mathrm{pg} / \mathrm{ml}, \mathrm{IQR}_{\mathrm{AN}}=10.8-30.7$, median $_{\mathrm{AN}-\mathrm{REC}}=9.2 \mathrm{pg} / \mathrm{ml}$, $\mathrm{IQR}_{\mathrm{AN}-\mathrm{REC}}=5.1-15.9$, and $p=0.005$. Replication sample: $\operatorname{median}_{\mathrm{AN}}=15.6 \mathrm{pg} / \mathrm{ml}, \mathrm{IQR}_{\mathrm{AN}}=12.1-21.3$, median $_{\mathrm{AN}}$ $\mathrm{REC}=11.1 \mathrm{pg} / \mathrm{ml}, \mathrm{IQR}_{\mathrm{AN}-\mathrm{REC}}=8.6-15.5$, and $p=1.64$. $10^{-6}$ ) and with CTRLs (Discovery sample: median $_{\mathrm{CTRL}}=$ $7.8 \mathrm{pg} / \mathrm{ml}, \mathrm{IQR}_{\mathrm{CTRL}}=4.7-9.9$, and $p=0.005$. Replication sample: $\operatorname{median}_{\mathrm{CTRL}}=9.3 \mathrm{pg} / \mathrm{ml}, \quad \mathrm{IQR}_{\mathrm{CTRL}}=6.4-12.9$, and $p=1.16 \cdot 10^{-13}$ ). The levels in AN-REC were significantly higher than CTRLs only in the larger replication sample $\left(p_{\text {discovery }}=0.967\right.$ and $\left.p_{\text {replication }}=0.004\right)$ (Fig. 2, see Table 2 for results of the linear model). Plasma NfL levels were negatively associated with BMI across all samples $\left(\beta \quad( \pm \mathrm{se})=-0.62 \pm 0.087\right.$ and $\left.p=6.9 \cdot 10^{-12}\right)$. However, the slopes were significantly different in the AN group compared with the AN-REC and CTRL groups $(p=0.022$ and 0.018 , respectively). No significant difference in slopes was seen when comparing AN-REC with CTRL $(p=0.957)$ (Fig. 3).

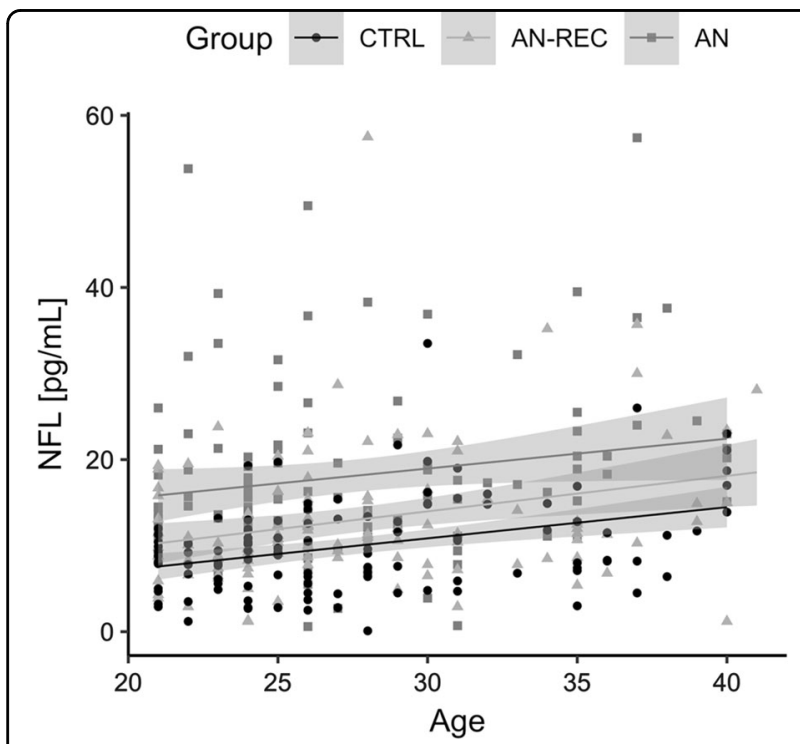

Fig. 1 Plasma neurofilament light (NfL) levels are correlated with age in individuals with anorexia nervosa (AN), weight recovered from AN (AN-REC), and normal-weight healthy controls (CTRL). Overall age effect, $\beta( \pm \mathrm{se})=0.37 \pm 0.087$ and $p=2.7 \cdot 10^{-5}$. The shaded line around each linear fit line represents $95 \%$ confidence interval
Finally, an association between the years since $\mathrm{AN}$ onset and NfL levels could be detected $(\beta( \pm \mathrm{se})=0.31 \pm 0.11$ and $p=0.006$ ), but this association did not withstand correcting for age.

\section{Discussion}

Here, we demonstrate significantly higher plasma levels of a marker for acute neuronal injury, $\mathrm{NfL}^{24-26}$, in female patients with AN, compared with normal-weight females with and without a history of AN. The difference in plasma NfL between AN-REC and CTRLS was smaller than the difference between AN and CTRLS suggesting that NfL might normalize somewhat with weight recovery. Elevated NfL levels have previously been documented in blood and/or CSF in several neurodegenerative conditions $^{32-36}$, in ischaemic stroke ${ }^{37}$, and even in bipolar disorder ${ }^{38}$.

As to the origin of the neuronal injury proposed by the elevated NfL levels in AN, we can only speculate. Ehrlich et al. evaluated the levels of other blood markers related to neuronal or glial damage in AN: neuron-specific enolase, glial fibrillary acidic protein (GFAP) and S100B, in AN blood $^{23,39}$. Contrary to our NfL findings, none of these three markers were altered in patients with active $\mathrm{AN}$ or recovered from $\mathrm{AN}$, which might be explained by the substantially smaller number of study subjects in these former studies. Imaging studies of individuals with AN have, however, also yielded heterogeneous results. While several studies have documented structural grey matter or WM changes both globally and focally in AN, many but not all of these changes appear to reverse upon recovery ${ }^{10}$. Since NfL is predominantly increased upon axonal injury, WM is the primary suspect source of origin ${ }^{26}$. The list of areas in which structural WM reductions have been documented in AN includes, but is not limited to, the dorsal striatum ${ }^{40}$, the hippocampus ${ }^{41}$, the hippocampalamygdala formation ${ }^{42}$, and the thalamus ${ }^{43}$. By diffusion tensor imaging and subsequent tractography analysis Florent and colleagues show reduced thickness of fibre tracts in a key food intake regulating hypothalamic area in AN compared with healthy normal weight and constitutionally lean controls (Florent, Baroncini, JissendiTchofo, Lopes, Vanhoutte, Rasika, Pruvo, Vignau, Verdun, Johansen, Pigeyre, Bouret, Nilsson and Prevot, in preparation). The hypothalamus is an area of the brain that among others is crucial for the regulation of food intake and body weight, i.e., energy homeostasis ${ }^{44}$, an area in which signs of degeneration have been documented in the spontaneously anorectic anx/anx mouse ${ }^{45}$. Furthermore, reduced connectivity between the orbitofrontal cortex and amygdala to the hypothalamus ${ }^{46}$, as well as focal grey matter atrophy in the hypothalamus has been documented in $\mathrm{AN}^{47}$. It is tempting to speculate that degeneration of hypothalamic neurocircuitries 

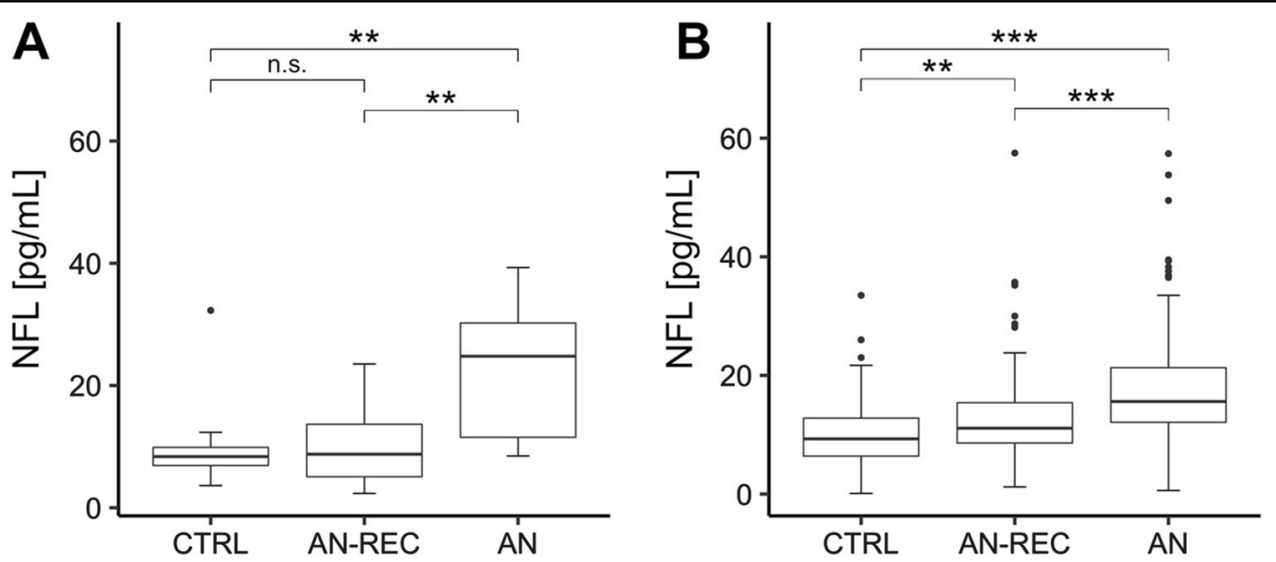

Fig. 2 Box plot graphs showing plasma levels of neurofilament light ( $N f L$ ) in individuals with anorexia nervosa (AN), weight recovered from AN (ANREC) and normal-weight healthy controls (CTRL) in the discovery cohort (a) and the replication cohort (b). The median is shown as a straight line and the box denotes the interquartile range. $P$-values corrected for age, ${ }^{* *} p<0.01$ and ${ }^{* *} p<0.001$

Table 2 Results from linear regression analyses of plasma neurofilament light ( $\mathrm{NfL}$ ) levels and effects of group and age in the replication cohort

\begin{tabular}{lllll}
\hline Independent variables & Estimate $(\boldsymbol{\beta})$ & SE & $\boldsymbol{t}$ & $\boldsymbol{p}$ \\
\hline Model 1 (adjusted $R^{2}=0.1906$, ANOVA: $F$ & $27.52, n=339, p=6.19 \mathrm{e}-16$ ) \\
(intercept) & -0.356 & 2.340 & -0.152 & 0.879 \\
Group AN-REC & 3.026 & 1.048 & 2.887 & 0.004 \\
Group AN & 8.153 & 1.053 & 7.743 & $1.16 \mathrm{e}-13$ \\
Age at sample & 0.374 & 0.080 & 4.683 & $4.11 \mathrm{e}-06$ \\
\hline
\end{tabular}

$A N$ anorexia nervosa, $A N-R E C$ recovered from anorexia nervosa

responsible for energy homeostasis are involved in the paradoxical response to underweight seen in AN, i.e., the perpetual low food intake. In fact, AN genome wide association study have yielded significant negative singlenucleotide-based genetic correlations with BMI and other anthropometric measures ${ }^{48-50}$ which mechanistically could have its origin in the hypothalamus.

Another possibility is also that the elevated NfL levels in AN reflects a global reduction in brain volume as indicated by enlarged ventricles or CSF spaces/volumes and sulcal widening ${ }^{10,12,18,21,51-55}$, or even a peripheral neuropathy that might occur due malnutrition-related thiamine deficiency ${ }^{56,57}$.

The levels of NfL were negatively associated with BMI in AN, AN-REC, and CTRLS, but the slope was much steeper in the underweight BMI range/AN group. This is consistent with the findings that many of the structural brain differences reverse with recovery/normalization of body weight ${ }^{10}$, and have even been shown to correlate with weight $\operatorname{loss}^{53}$. Future studies should explore the levels of NfL in constitutionally lean individuals, in order

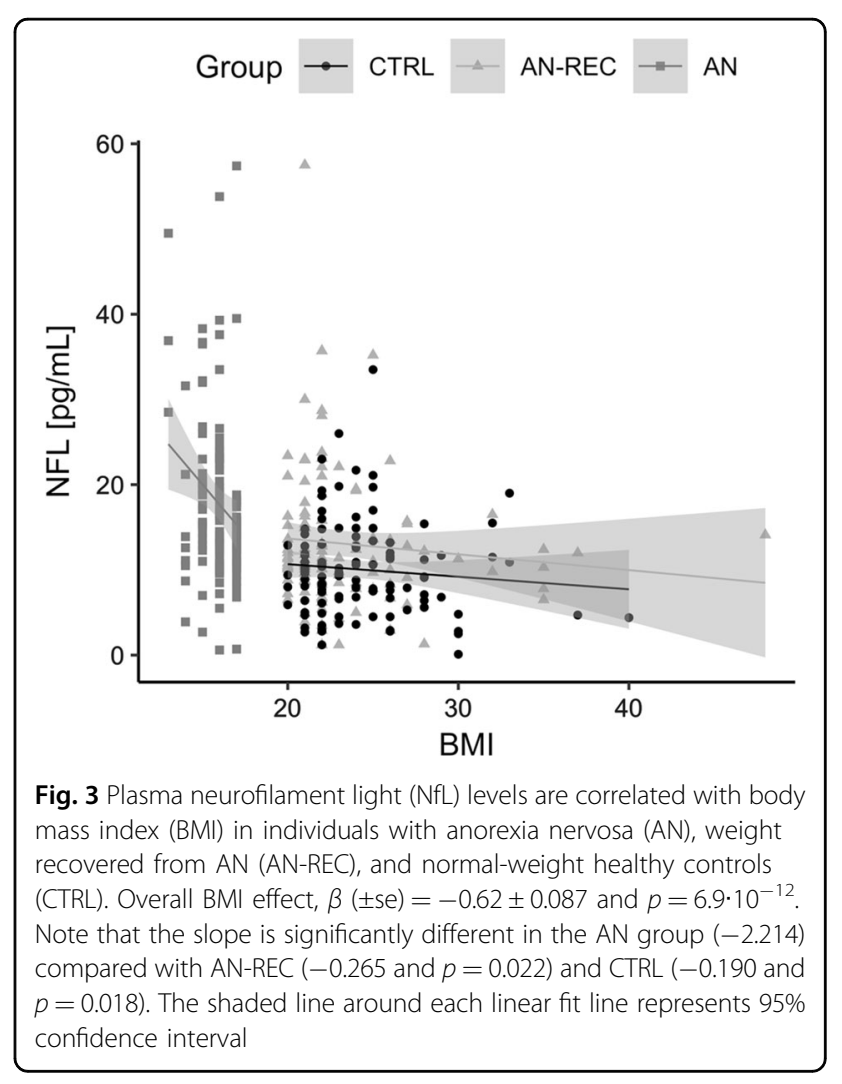

to clarify if the increased plasma NfL in AN is exclusively an effect of low BMI.

One limitation of this study is that the levels of NfL were not compared with synchronized imaging in the same individuals. Another limitation is that since NfL levels reflect ongoing neuronal injury or degenerative process, we cannot determine the long-term and potentially permanent effects of the processes indicated by such 
increased levels. The difference in plasma NfL between AN-REC and CTRLS is smaller than the difference between AN and CTRLS, which could indicate that the levels normalize with time of recovery. The minimum recovery time was 1 year in the present study. It would be of value to evaluate the levels of NFL in plasma from individuals recovered from AN for a longer period, e.g., 5 years. But even if the levels would normalize completely with a longer time since recovery, it is still possible that the neuronal injury observed during active AN or shortterm recovery has long-term structural and functional effects on the brain. Upregulation of brain derived neurotrophic factor has been documented in patients recovered from $\mathrm{AN}$, which might indicate a regenerative response to a neuronal injury during the prolonged starvation $^{58}$. Brain-imaging studies combined with long-term follow up evaluation of NfL levels are needed to clarify this.

Plasma and CSF levels of NfL are known to be very highly correlated ${ }^{28}$. It is however possible that the blood-brain barrier (BBB) becomes more permeable in starved conditions such as $\mathrm{AN}$, which allows more NfL to leak into the circulation. In fact, in mice the BBB becomes more permeable after fasting ${ }^{59}$. Future studies should include evaluation of NfL in CSF of AN patients in order to explore a potential effect of a leaky BBB in AN.

To conclude, we report for the first time that plasma NfL levels are significantly increased in individuals with AN, compared with AN-REC and CTRLs. The levels were lower in recovered than underweight AN patients indicating that the active neuronal injury/degenerative process might attenuate upon recovery.

\section{Acknowledgements \\ We are deeply grateful for the participation of all subjects contributing to this research. We thank the collection team that worked to recruit them, as well as data manager Bozenna lliadou. We also wish to thank the National Quality Registry for Eating Disorders (RIKSÄT) for help with recruiting patients. We finally wish to thank the BBMRl.se and KI Biobank at Karolinska Institutet for professional biobank service. Funding support was provided from the Stockholm County Council (ALF 20170019 and 20170058), the Swedish Brain Foundation, Karolinska Institutet (KI), the Swedish Research Council (\#2017- 00915, \#2018-02532 and \#2018-02653), the Swedish foundation for Strategic Research KF10-0039, the European Research Council (\#681712), Swedish State Support for Clinical Research (ALFGBG-715986 and ALFGBG-720931), and the Olav Thon Foundation. C.M.B. acknowledges funding from the Swedish Research Council (Vetenskapsrådet, award: 538-2013-8864). The Anorexia Nervosa Genetics Initiative (ANGI) is an initiative of the Klarman Family Foundation. H.Z. is a Wallenberg Academy Fellow. K.B. holds the Torsten Söderberg Professorship in Medicine at the Royal Swedish Academy of Sciences.}

\section{Author details}

'Department of Molecular Medicine and Surgery, Karolinska Institutet, Stockholm, Sweden. ${ }^{2}$ Center for Molecular Medicine, Karolinska University Hospital, Stockholm, Sweden. ${ }^{3}$ Centre for Eating Disorders Innovation, Karolinska Institutet, Stockholm, Sweden. ${ }^{4}$ Department of Clinical Neuroscience, Karolinska Institutet, Stockholm, Sweden. ${ }^{5}$ Tema Neuro, PO3, Karolinska University Hospital, Stockholm, Sweden. ${ }^{6}$ Department of Medical Epidemiology and Biostatistics, Karolinska Institutet, Stockholm, Sweden. ${ }^{7}$ Centre for Psychiatry Research, Department of Clinical Neuroscience,
Karolinska Institutet, Stockholm, Sweden. ${ }^{8}$ Stockholm Health Care Services, Stockholm County Council, Stockholm, Sweden. ${ }^{9}$ Stockholm Centre for Eating Disorders, Stockholm, Sweden. ${ }^{10}$ Department of Psychiatry and Neurochemistry, Institute of Neuroscience and Physiology, The Sahlgrenska Academy at the University of Gothenburg, Mölndal, Sweden. ${ }^{11}$ Clinical Neurochemistry Laboratory, Sahlgrenska University Hospital, Mölndal, Sweden. ${ }^{12}$ Department of Psychiatry, University of North Carolina at Chapel Hill, Chape Hill, NC, USA. ${ }^{13}$ Department of Nutrition, University of North Carolina at Chapel Hill, Chapel Hill, NC, USA. ${ }^{14}$ Department of Neurodegenerative Disease, UCL Queen Square Institute of Neurology, Queen Square, London, UK. ${ }^{15}$ UK Dementia Research Institute at UCL, London, UK

\section{Author contributions}

I.A.K.N., V.M., V.D.K., C.M.B., H.Z., and M.L., designed the study. I.A.K.N., A.J., C.N., Y. H.J., C.M.B., and M.L., collected the samples. K.B. and H.Z. performed the assay. I. A.K.N. and V.M., analysed the data. I.A.K.N., V.M., V.D.K., A.M.S., K.B., C.M.B., H.Z., and M.L. wrote the paper, which was revised and approved by all authors.

\section{Conflict of interest}

H.Z. has served at scientific advisory boards for Roche Diagnostics, Wave, Samumed, and CogRx, and is a co-founder of Brain Biomarker Solutions in Gothenburg AB, a GU Ventures-based platform company at the University of Gothenburg (all unrelated to the submitted work). M.L. declares that, over the past 36 months, he has received lecture honoraria from Lundbeck pharmaceutical. C.M.B. reports: Shire (grant recipient, Scientific Advisory Board member); Pearson and Walker (author, royalty recipient). No other equity ownership, profit-sharing agreements, royalties, or patent. V.D.K. reports: Stockholm County Council (grant recipient ALF 20160457); Biogen (recipient of grant and scholarship, PI for project sponsored by); Novartis (recipient of scholarship and lecture honoraria); Merc (Scientific Advisory Board member, recipient of lecture honoraria). K.B. has served as a consultant or at advisory boards for Alector, Alzheon, CogRx, Biogen, Lilly, Novartis, and Roche

Diagnostics, and is a co-founder of Brain Biomarker Solutions in Gothenburg $A B$, a GU Venture-based platform company at the University of Gothenburg (all unrelated to the work presented in this paper).

\section{Publisher's note}

Springer Nature remains neutral with regard to jurisdictional claims in published maps and institutional affiliations.

Received: 6 March 2019 Accepted: 20 June 2019

Published online: 01 August 2019

\section{References}

1. Schaumberg, K. et al. The science behind the academy for eating disorders' nine truths about eating disorders. Eur. Eat. Disord. Rev. 25, 432-450 (2017).

2. Association AP. Diagnostic and statistical manual of mental disorders (DSM-5) (American Psychiatric Publishing, Arlington, VA, 2013).

3. Papadopoulos, F. C., Ekbom, A., Brandt, L. \& Ekselius, L. Excess mortality, causes of death and prognostic factors in anorexia nervosa. Br. J. Psychiatry 194, 10-17 (2009).

4. Keski-Rahkonen, A. et al. Epidemiology and course of anorexia nervosa in the community. Am. J. Psychiatry 164, 1259-1265 (2007).

5. Bulik, C. M. et al. Prevalence, heritability, and prospective risk factors for anorexia nervosa. Arch. Gen. Psychiatry 63, 305-312 (2006).

6. Berends, T., Boonstra, N. \& van Elburg, A. Relapse in anorexia nervosa: a systematic review and meta-analysis. Curr. Opin. Psychiatry 31, 445-455 (2018).

7. Wade, T. D., Bulik, C. M., Neale, M. \& Kendler, K. S. Anorexia nervosa and major depression: shared genetic and environmental risk factors. Am. J. Psychiatry 157, 469-471 (2000).

8. Hoek, H. W. Incidence, prevalence and mortality of anorexia nervosa and other eating disorders. Curr. Opin. Psychiatry 19, 389-394 (2006).

9. King J. A., Frank G. K. W., Thompson P. M., Ehrlich S. Structural neuroimaging of anorexia nervosa: future directions in the quest for mechanisms underlying dynamic alterations. Biol. Psychiatry. 83, 224-234 (2018).

10. Phillipou, A., Rossell, S. L. \& Castle, D. J. The neurobiology of anorexia nervosa: a systematic review. Aust. N. Z. J. Psychiatry 48, 128-152 (2014). 
11. Titova, O. E., Hjorth, O. C., Schioth, H. B. \& Brooks, S. J. Anorexia nervosa is linked to reduced brain structure in reward and somatosensory regions: a metaanalysis of VBM studies. BMC Psychiatry 13, 110 (2013).

12. Castro-Fornieles, J. et al. A cross-sectional and follow-up voxel-based morphometric MRI study in adolescent anorexia nervosa. J. Psychiatr. Res. 43, 331-340 (2009)

13. Muhlau, M. et al. Gray matter decrease of the anterior cingulate cortex in anorexia nervosa. Am. J. Psychiatry 164, 1850-1857 (2007).

14. King, J. A. et al. Global cortical thinning in acute anorexia nervosa normalizes following long-term weight restoration. Biol. Psychiatry 77, 624-632 (2015).

15. Bernardoni, F. et al. Weight restoration therapy rapidly reverses cortical thinning in anorexia nervosa: a longitudinal study. Neuroimage 130, 214-222 (2016).

16. Roberto, C. A. et al. Brain tissue volume changes following weight gain in adults with anorexia nervosa. Int. J. Eat. Disord. 44, 406-411 (2011).

17. McCormick, L. M. et al. Implications of starvation-induced change in right dorsal anterior cingulate volume in anorexia nervosa. Int. J. Eat. Disord. 41, 602-610 (2008).

18. Kingston, K., Szmukler, G., Andrewes, D., Tress, B. \& Desmond, P. Neuropsychological and structural brain changes in anorexia nervosa before and after refeeding. Psychol. Med. 26, 15-28 (1996).

19. Lazaro, L. et al. Normal gray and white matter volume after weight restoration in adolescents with anorexia nervosa. Int. J. Eat. Disord. 46, 841-848 (2013).

20. Bang, L., Ro, O. \& Endestad, T. Normal white matter microstructure in women long-term recovered from anorexia nervosa: a diffusion tensor imaging study. Int. J. Eat. Disord. 51, 46-52 (2018).

21. Dolan, R. J., Mitchell, J. \& Wakeling, A. Structural brain changes in patients with anorexia nervosa. Psychol. Med. 18, 349-353 (1988).

22. Shott, M. E., Pryor, T. L., Yang, T. T. \& Frank, G. K. Greater insula white matter fiber connectivity in women recovered from anorexia nervosa. Neuropsychopharmacology 41, 498-507 (2016).

23. Ehrlich, S. et al. S100B in underweight and weight-recovered patients with anorexia nervosa. Psychoneuroendocrinology 33, 782-788 (2008).

24. Ljungqvist, J., Zetterberg, H., Mitsis, M., Blennow, K. \& Skoglund, T. Serum neurofilament light protein as a marker for diffuse axonal injury: results from a case series study. J. Neurotrauma 34, 1124-1127 (2017).

25. Zetterberg, H. Neurofilament light: a dynamic cross-disease fluid biomarker for neurodegeneration. Neuron 91, 1-3 (2016).

26. Khalil, M. et al. Neurofilaments as biomarkers in neurological disorders. Nat. Rev. Neurol. 14, 577-589 (2018).

27. Thornton, L. M. et al. The anorexia nervosa genetics initiative (ANGI): overview and methods. Contemp. Clin. Trials 74, 61-69 (2018).

28. Gisslen, M. et al. Plasma concentration of the neurofilament light protein (NFL) is a biomarker of CNS injury in HIV infection: a cross-sectional study. EBioMedicine 3, 135-140 (2016).

29. Hothorn, T., Bretz, F. \& Westfall, P. Simultaneous inference in general parametric models. Biom. J. 50, 346-363 (2008).

30. Wickham H. ggplot2: Elegant Graphics for Data Analysis. New York: SpringerVerlag (2016).

31. Lewczuk, P. et al. Plasma neurofilament light as a potential biomarker of neurodegeneration in Alzheimer's disease. Alzheimer's Res. Ther. 10, 71 (2018).

32. Rohrer, J. D. et al. Serum neurofilament light chain protein is a measure of disease intensity in frontotemporal dementia. Neurology 87, 1329-1336 (2016).

33. Meeter, L. H. et al. Neurofilament light chain: a biomarker for genetic frontotemporal dementia. Ann. Clin. Transl. Neurol. 3, 623-636 (2016).

34. Kovacs, G. G. et al. Plasma and cerebrospinal fluid tau and neurofilament concentrations in rapidly progressive neurological syndromes: a neuropathology-based cohort. Eur. J. Neurol. 24, 1326-e77 (2017).

35. Bergman, J. et al. Neurofilament light in CSF and serum is a sensitive marker for axonal white matter injury in MS. Neurol. Neuroimmunol. Neuroinflamm. 3, e271 (2016).

36. Ashton, N. J. et al. Increased plasma neurofilament light chain concentration correlates with severity of post-mortem neurofibrillary tangle pathology and neurodegeneration. Acta Neuropathol. Commun. 7, 5 (2019).
37. Pujol-Calderon F. et al. Neurofilament changes in serum and cerebrospinal fluid after acute ischemic stroke. Neurosci. Lett. 698, 58-63 (2019).

38. Jakobsson, J. et al. Elevated concentrations of neurofilament light chain in the cerebrospinal fluid of bipolar disorder patients. Neuropsychopharmacology 39, 2349-2356 (2014).

39. Ehrlich, S. et al. Glial and neuronal damage markers in patients with anorexia nervosa. J. Neural Transm. 115, 921-927 (2008).

40. Frank, G. K., Shott, M. E., Hagman, J. O. \& Mittal, V. A. Alterations in brain structures related to taste reward circuitry in ill and recovered anorexia nervosa and in bulimia nervosa. Am. J. Psychiatry 170, 1152-1160 (2013).

41. Connan, F. et al. Hippocampal volume and cognitive function in anorexia nervosa. Psychiatry Res. 146, 117-125 (2006).

42. Giordano, G. D. et al. Volume measurement with magnetic resonance imaging of hippocampus-amygdala formation in patients with anorexia nervosa. J. Endocrinol. Investig. 24, 510-514 (2001).

43. Frieling, $\mathrm{H}$. et al. Microstructural abnormalities of the posterior thalamic radiation and the mediodorsal thalamic nuclei in females with anorexia nervosa - a voxel based diffusion tensor imaging (DTI) study. J. Psychiatr. Res. 46, 1237-1242 (2012).

44. Schwartz, M. W. Central nervous system regulation of food intake. Obesity 14 1S-8S (2006).

45. Nilsson, I. A. et al. Evidence of hypothalamic degeneration in the anorectic anx/anx mouse. Glia 59, 45-57 (2011).

46. Frank, G. K., Shott, M. E., Riederer, J. \& Pryor, T. L. Altered structural and effective connectivity in anorexia and bulimia nervosa in circuits that regulate energy and reward homeostasis. Transl. psychiatry 6, e932 (2016).

47. Boghi, A. et al. In vivo evidence of global and focal brain alterations in anorexia nervosa. Psychiatry Res. 192, 154-159 (2011).

48. Duncan, L. et al. Significant locus and metabolic genetic correlations revealed in genome-wide association study of anorexia nervosa. Am. J. Psychiatry 174, 850-858 (2017)

49. Hinney, A. et al. Evidence for three genetic loci involved in both anorexia nervosa risk and variation of body mass index. Mol. Psychiatry 22, 321-322 (2017).

50. Watson, H. J. et al. Genome-wide association study identifies eight risk loci and implicates metabo-psychiatric origins for anorexia nervosa. Nat Genet. (2019). https://doi.org/10.1038/s41588-019-0439-2. [Epub ahead of print].

51. Katzman, D. K., Zipursky, R. B., Lambe, E. K. \& Mikulis, D. J. A longitudinal magnetic resonance imaging study of brain changes in adolescents with anorexia nervosa. Arch. Pediatr. Adolesc. Med. 151, 793-797 (1997).

52. Katzman, D. K. et al. Cerebral gray matter and white matter volume deficits in adolescent girls with anorexia nervosa. J. Pediatr. 129, 794-803 (1996).

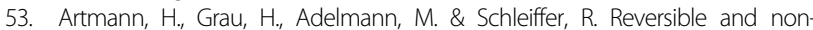
reversible enlargement of cerebrospinal fluid spaces in anorexia nervosa. Neuroradiology 27, 304-312 (1985).

54. Palazidou, E., Robinson, P. \& Lishman, W. A. Neuroradiological and neuropsychological assessment in anorexia nervosa. Psychol. Med. 20, 521-527 (1990).

55. Swayze, V. W. 2nd et al. Reversibility of brain tissue loss in anorexia nervosa assessed with a computerized Talairach 3-D proportional grid. Psychol. Med. 26, 381-390 (1996).

56. Renthal, W., Marin-Valencia, I. \& Evans, P. A. Thiamine deficiency secondary to anorexia nervosa: an uncommon cause of peripheral neuropathy and Wernicke encephalopathy in adolescence. Pediatr. Neurol. 51, 100-103 (2014).

57. MacKenzie, J. R., LaBan, M. M. \& Sackeyfio, A. H. The prevalence of peripheral neuropathy in patients with anorexia nervosa. Arch. Phys. Med. Rehabil. 70, 827-830 (1989).

58. Ehrlich, S. et al. Serum brain-derived neurotrophic factor and peripheral indicators of the serotonin system in underweight and weight-recovered adolescent girls and women with anorexia nervosa. J. Psychiatry Neurosci. 34, 323-329 (2009).

59. Langlet, F. et al. Tanycytic VEGF-A boosts blood-hypothalamus barrier plasticity and access of metabolic signals to the arcuate nucleus in response to fasting. Cell Metab. 17, 607-617 (2013). 